\title{
YO Y LA ECONOMÍA. EVALUACIÓN DE LA ACTUACIÓN DOCENTE EN UN PROGRAMA DE EDUCACIÓN ECONÓMICA PARA ESCOLARES DE 6. ${ }^{\text {to }}$ AÑO DE EDUCACIÓN PRIMARIA
}

\author{
Me and the economy. Evaluation of the performance in a teacher economic \\ education program for schoolchildren in $6 .{ }^{\text {th }}$ grade \\ Marianela Denegri C. ${ }^{1}$, Carlos Del Valle R., Soledad Etchebarne L., \\ Yéssica González G., Jocelyne Sepúlveda A. \\ Universidad de La Frontera, Temuco, Chile \\ (Recibido el 08/03/2011 - Aceptado el 09/06/2011)
}

\begin{abstract}
RESUMEN
La presente investigación estudió el cambio en las prácticas pedagógicas de profesores y profesoras de Educación Primaria, a partir de su participación en un Programa de formación en Educación Económica para escolares de $6 .^{\text {to }}$ año. La muestra estuvo conformada por 14 profesores de las asignaturas de Comprensión del Medio, Lenguaje y Comunicación y Matemáticas, a quienes se aplicó el Test de Alfabetización Económica para Adultos (TAE-A) (Gempp, Denegri, Catalán, Caripán, Hermosilla y Caprile, 2007) y la Pauta de Observación de Práctica Docentes (Denegri, 2005). Los resultados revelan un aumento en los niveles de alfabetización económica y diferencias significativas en la práctica docente de las y los profesores participantes, hallazgos que se discuten a partir de los antecedentes bibliográficos.
\end{abstract}

Palabras clave: Educación económica, alfabetización económica, programa de formación, profesores.

\begin{abstract}
The present research studied the change in teaching practices of Basic Education (Primary School) teachers, from their participation in a training program in Economic Education. The sample consisted in 14 teachers of Understanding of the Environment, Language and Communication, and Mathematics courses, to who were applied the Economic Literacy Test for Adults (TAE-A) (Gempp, Denegri, Catalan, Caripán, Hermosilla \& Caprile, 2007) and the Pattern of Observation of Teaching Practices (Denegri, 2005). The results reveals an increase in literacy economic levels and significant differences in teaching practices of the teachers participants, results are discussed considering the bibliographic review.
\end{abstract}

Keywords: Economic education, economic literacy, training program, teachers.

1 Docente investigadora del Centro de Excelencia en Psicología Económica y del Consumo, Universidad de La Frontera, Chile. E-mail: mdenegri@ufro.cl 


\section{INTRODUCCIÓN}

Uno de los objetivos fundamentales del desarrollo en América Latina debe ser romper el círculo vicioso que vincula un bajo crecimiento con pobreza, inequidad distributiva y exclusión social, lo que implica la necesidad de desarrollar nuevos modelos de desarrollo económico y reducir la pobreza y las brechas entre los distintos sectores de la población, potenciando la posibilidad de progresar socialmente gracias al acceso a distintos activos, entre los cuales la educación reviste especial importancia (Machinea, 2007). En este sentido, superar la pobreza y la desigualdad, reformar la educación y diversificar la economía son objetivos presentes en la agenda de desarrollo de la mayoría de los países latinoamericanos (Cumbre Extraordinaria de las Américas, 2004). Estos propósitos, sin embargo, contrastan con las capacidades reales de las personas para responder a los crecientes requerimientos de sociedades más complejas. Un aspecto crítico de estas habilidades está vinculado con el desarrollo de destrezas y actitudes que permitan regular la conducta personal y colectiva hacia un uso racional de recursos económicos escasos, y por ello la preparación real que poseen los ciudadanos para enfrentarse a un mercado creciente que presiona hacia el consumo en un contexto medio ambiental en constante deterioro (Volpe, Haiyang y Sheen, 2006).

En este contexto, en Chile, el Informe de la Comisión de Formación Ciudadana (2004) señala que la formación ciudadana debe entregar herramientas fundamentales de análisis económico que permitan manejar adecuadamente los desafíos que impone la realidad. Es decir, acceder a un nivel eficiente de alfabetización económica que permita a los ciudadanos el manejo adecuado de conceptos económicos básicos, administrar adecuadamente los recursos económicos personales y familiares, tomar decisiones que optimicen el uso de dichos recursos y proyectar su situación financiera en el futuro comprendiendo las consecuencias que tiene el tema de la previsión y su adecuada comprensión (Lusardi y Mirchelli, 2007).

Sin embargo, numerosos estudios sobre el desarrollo de competencias económicas básicas en la población, tanto a nivel mundial como chilena, constatan las serias dificultades que presentan adolescentes y adultos, tanto para comprender la economía cotidiana como para actuar eficientemente en ella; lo cual redunda en problemas económico-sociales masivos como el sobreendeudamiento, el consumismo y la mala planificación de los recursos familiares y personales, lo que se agrava aún más en el caso de los sectores más pobres. Los mismos estudios indican que factores como escolarización, ciudad de residencia, género y nivel socioeconómico inciden en la manera que niños, adolescentes y adultos comprenden los fenómenos económicos (Denegri y Palavecinos, 2003; Gleason y Van Scyoc, 1995; Johnston y Maguire, 2004; Lusardi y Mitchelli, 2007; Medina, Méndez y Pérez, 1999). El problema es aún más serio si se considera que el analfabetismo económico se reproduce a través de la socialización económica en la familia (Denegri, Palavecinos, Ripoll y Yáñez, 1999; Moore-Shay y Berchmans, 1996; Page y Ridgway, 2001; Rose, 1999); donde al carecer los propios padres de una adecuada alfabetización económica (Mori, 2001) se produce una transmisión intergeneracional de pautas de consumo y uso del dinero poco reflexivas o ineficientes (Denegri, Palavecinos y Gempp, 2005). 
Este déficit en educación económica en la infancia se mantiene hasta la adolescencia y la adultez, no observándose que el sistema educativo logre compensarlos. Ello debido, en parte, a la poca extensión, 20 a 22 semanas de los 12 años de escolarización, y organización de la alfabetización económica en el currículum, ya que las temáticas aparecen en forma aislada en distintos momentos y unidades, sin un eje que las aglutine, y sin un modelo pedagógico (Domper, 2004).

La mayoría de los estudios en relación a los modelos de educación económica coinciden en que es fundamental incorporar la relación entre al menos tres variables que interactúan en el comportamiento económico, ellas son: (a) las elecciones económicas, (b) los conceptos económicos y (c) las metas del comportamiento, enfatizándose la importancia de una aproximación interdisciplinaria a la enseñanza de conceptos económicos. En varios de estos trabajos se señala que los conceptos básicos que deberían ser abordados son: (a) escasez y recursos limitados, (b) costo de oportunidad y análisis de costo-beneficio en la elección de alternativas, (c) mercado y producción de bienes y servicios, (d) funcionamiento bancario, (e) consumo e ingresos, (f) dinero y formas de intercambio, (g) distribución de recursos y (h) oferta y demanda (Alemayehu, 2007; Kourlinsky, 1996; Laney, 1993; Mandell, 2008; Orton, 2007; Schug, 1996; Schug y Hagedorn, 2005; Sosin, Dick y Lynn, 1997; Walstad y Rebeck, 2001).

En cuanto a la efectividad de los programas sistemáticos de educación económica incorporados al curriculum (Georgius, 1996; Moos, 2007; Sossin et al., 1997; Varcoe y Fitch, 2003; Wentland, 2004), los resultados concuerdan en que el aprendizaje muestra tener un alto impacto no sólo en el nivel de conocimientos sino también en la conducta cotidiana de los estudiantes.

Por otra parte, los estudios concluyen que los profesores de escuelas primarias y secundarias, si bien poseen entrenamiento y experiencia en el desarrollo de estrategias pedagógicas, raramente tienen una preparación en temas económicos. La formación sistemática que incorpore evaluación de su propio nivel de alfabetización económica, aprendizaje de conceptos claves, reflexión sobre sus propios comportamientos económicos y acompañamiento en la implementación de estrategias de educación económica, tiene un importante impacto en el cambio de sus metodologías pedagógicas y por ende en los niveles de aprendizaje de los alumnos (Kourilsky, 1996; Moos, 2007; Parkinson, Sorgman y Millar, 2004; Vargha, 2004; Walstad y Allgood, 1999; Walstad y Rebeck, 2001).

\section{Nuestra propuesta: El programa Yo y la Economía}

El programa tiene por propósito empoderar a los estudiantes de $6 .{ }^{\text {to }}$ año de Educación General Básica para construir aprendizajes significativos a través de una conexión generativa entre los contextos de la economía familiar cotidiana y el entorno económico externo de tal forma que comprendan conceptos básicos de economía, desarrollen estrategias de razonamiento crítico y de toma de decisiones y actitudes proactivas que sirvan de sustento a conductas de emprendimiento personal y social (Denegri, Del Valle, Gempp y Lara, 2006a).

A través del programa de Educación Económica Yo y la Economía, mediante una aproximación interdisciplinaria integra sectores de Estudio y Comprensión del Medio 
Social, Matemáticas y Lenguaje y Comunicación, enseña conceptos económicos orientados al desarrollo de conocimientos y competencias económicas vinculadas a las capacidades cognitivas de los niños (Denegri y Martínez, 2003). En forma previa al desarrollo del programa en aula con los escolares, se realiza una etapa de capacitación y entrenamiento para los profesores participantes que forma parte de un programa global de formación y acompañamiento (Denegri et al., 2006a; Denegri, Gempp, Del Valle, Etchebarne y González, 2006b).

Considerando aportes de la literatura (Laney, 1993; Moos; 2007; National Council on Economic Education, 1997), el esquema del programa de educación económica se organiza en torno a la toma de decisiones en un contexto de necesidades múltiples y recursos escasos, articulando en torno a ello el abordaje de siete conceptos económicos críticos, tal como se representa en la Figura 1.

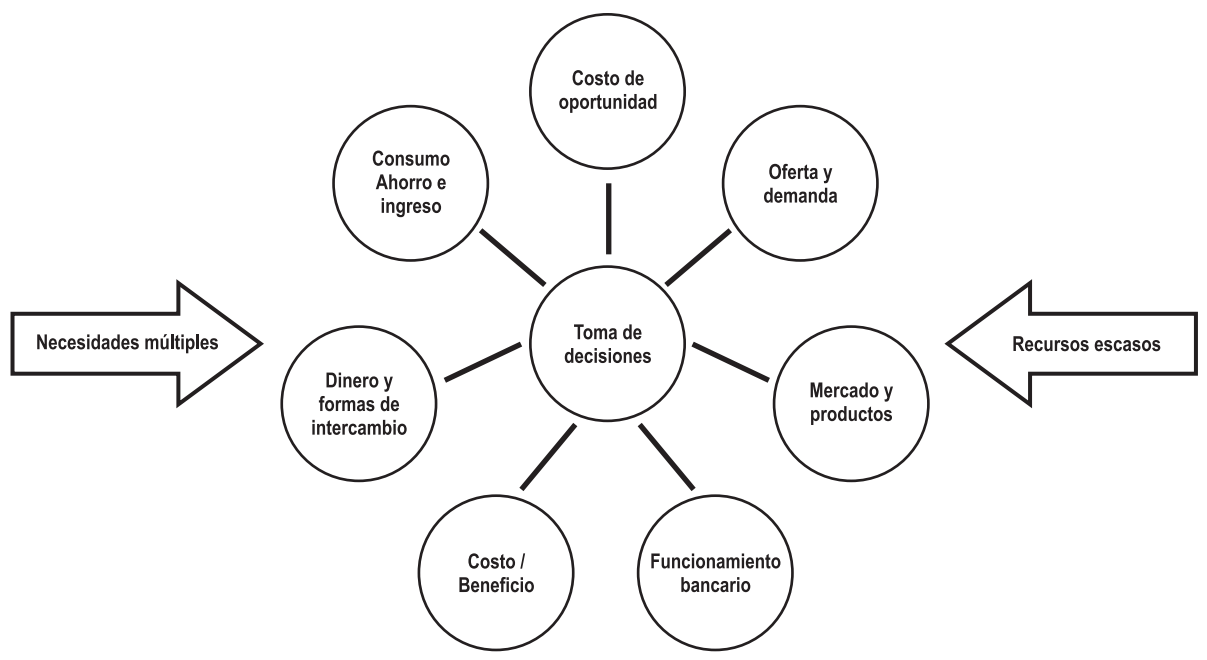

FIGURA 1. Conceptos básicos articuladores de un Modelo de Alfabetización Económica para alumnos de $6 .{ }^{\text {to }}$ año básico.

Fuente: elaboración propia.

Cada bloque temático se desarrolla considerando la presencia de cuatro tipos de actividades pedagógicas claves (Denegri et al., 2006a; 2006b): 1) Experiencia: estudiantes vivencian experiencialmente problemas y simulaciones de situaciones económicas complementadas con contenidos de la unidad didáctica; 2) Cuestionamiento: profesor cuestiona incitando la reflexión, aclarando conceptos de la experiencia y respondiendo preguntas; es el momento de la Alfabetización Económica Conceptual; 3) Refuerzo mediante la reflexión guiada: profesor profundiza reflexión y refuerza conceptos, guía el trabajo atendiendo dificultades y al mismo tiempo potencia la autonomía mediante la retroalimentación y establece la relación entre los distintos contenidos, articulando así el aporte de cada subsector. Aquí cobran importancia el uso de los medios tecnológicos y recursos propios de la cultura cotidiana de los niños (cómics, video juegos, dibujos animados, etc.); 4) Aplicación y ejercicio de competencias: desarrollo de un pequeño emprendimiento colectivo que puede 
ser de generación de recursos monetarios o de inversión social en trabajo con la comunidad articulado en torno al concepto de escasez.

De acuerdo a lo expuesto, los objetivos de esta investigación fueron, (a) describir el nivel de alfabetización económica de los profesores participantes de la muestra, antes de iniciar el programa de capacitación en educación económica (pre-test); (b) comparar los cambios en los niveles de alfabetización económica de los profesores luego de ser formados en el Programa (post-test), y (c) comparar los cambios en la actuación docente al inicio, durante y al término de la aplicación experimental del programa de educación económica Yo y la Economía.

\section{MÉTODO}

\section{Participantes}

Se seleccionó una muestra bietápica. En la primera etapa, de carácter no probabilístico e intencionado, se seleccionó un conjunto de establecimientos en base a criterios de inclusión/exclusión del proyecto (Denegri et al., 2006a), los que posteriormente fueron divididos en tres grupos según dependencia: Municipal, Particular Subvencionado y Particular Pagado. En la segunda etapa, de tipo probabilística simple, se seleccionaron al azar dos establecimientos de cada tipo de dependencia, y se les invitó a participar del estudio. En aquellos casos que los establecimientos declinaron su participación, se procedió a realizar una nueva selección azarosa del conjunto pre-seleccionado. Seleccionados los establecimientos participantes, la muestra constitutiva de esta investigación quedó conformada por 14 profesores de los sectores de Lenguaje y Comunicación, Comprensión del Medio Social y Matemáticas.

\section{Instrumentos}

Test de Alfabetización Económica para Adultos (TAE-A). Evalúa el nivel de comprensión de conceptos y prácticas económicas, posee un formato autoadministrado y con 23 ítems de selección múltiple, con una opción correcta. El instrumento fue calibrado con el Modelo de Rasch en una muestra de 840 adultos, mostrando un ajuste satisfactorio y adecuadas propiedades psicométricas (Alpha $=0.85)($ Gempp, Denegri, Catalán, Caripán, Hermosilla y Caprile, 2007).

Pauta de Observación de Práctica Docente. Desarrollada en estudios previos (Denegri, 2005) y adaptada para evaluar la actuación docente en el desarrollo de proyectos de aula en educación económica. Consta de 9 ítems puntuados en una escala de 1 (ausencia) a 3 (presencia total), que abordan dimensiones claves vinculadas a la construcción de aprendizajes significativos y que pueden actuar como facilitadores u obstaculizadores del desarrollo de proyectos educativos.

\section{Procedimiento}

Una vez seleccionados los establecimientos, fueron informados de los propósitos generales del Programa y se invitó a los docentes a participar en un curso de 120 hrs. de capacitación, 
dividido en una etapa de formación en aula y una de aplicación y desarrollo de proyectos de aula interdisciplinarios en educación económica. La aplicación del Test de Alfabetización Económica se realizó previo al inicio y una vez finalizada la capacitación; en tanto que la Pauta de Observación de Práctica Docente fue aplicada al inicio, proceso y final de la implementación de los proyectos de aula interdisciplinarios, en la segunda parte de la capacitación. En todo momento fue asegurada la confidencialidad de los datos y solicitadas las autorizaciones correspondientes para poder llevar a cabo la presente investigación.

\section{RESULTADOS}

La presentación de los resultados se desarrolla en torno a dos ejes temáticos. El primero corresponde a los cambios en los niveles de Alfabetización Económica y el segundo a los cambios observados en la práctica pedagógica durante el desarrollo de los proyectos en cada escuela por cada equipo docente.

\section{Alfabetización económica}

En la evaluación inicial se obtuvieron puntajes entre 7 y 17 puntos, con una media de 10,285 (d.s $=2,61)$, logrando un $44 \%$ de rendimiento, apreciándose importantes diferencias entre las distintas áreas de la economía que evalúa el test, el mejor desempeño correspondió a economía general, en un nivel medio bajo se encontraba microeconomía y claramente disminuido el rendimiento en macroeconomía y economía internacional.

En la evaluación pos-test los profesores presentan un aumento sustancial de su rendimiento ubicándose en un $77,25 \%$ de éxito, lo que indica un aumento en su rendimiento de más de un $30 \%$, con una media de 17,77 (d.s $=2,45)$. Al analizar las pruebas por sus tópicos más relevantes destaca, que las principales alzas se obtuvieron en las tres áreas que habían conseguido un rendimiento más bajo, manteniéndose estables los resultados obtenidos en economía general.

\section{Actuación docente en el desarrollo de proyectos de aula}

Como ya fue descrito, la actuación docente fue evaluada a partir de la aplicación de la Pauta de observación de la práctica docente, registrándose un total de 39 observaciones de aula válidas, cada una de las cuales tuvo una duración de 180 minutos. En relación a las 39 observaciones, 12 se efectuaron al inicio, 13 durante el proceso de implementación del Programa Yo y la Economía y 14 en la etapa final del programa, distribuidas según la dependencia de los establecimientos.

Al revisar los resultados del análisis de varianza (ver Tabla 1), es posible concluir que la actuación docente, evaluada a partir de los indicadores, presenta diferencias significativas respecto al momento de desarrollo de los proyectos de aula en los siguientes indicadores: evalúa conocimientos previos $(F=6,035, \mathrm{p}<0.01)$, hace preguntas de reflexión $(F=18,957, \mathrm{p}<0.01)$, relaciona contenidos $(F=34,728, \mathrm{p}<0.01)$, guía el trabajo de los alumnos $(F=10,821, \mathrm{p} \leq 0.01)$, retroalimenta el desempeño $(F=40,094, \mathrm{p}<0.01)$ y utiliza metodologías cooperativas $(F=20,862$, p $<0.01)$. Del total de los indicadores sólo tres de ellos (explicita objetivos, responde preguntas y atiende dificultades) no presentaron 
diferencias significativas respecto al momento de ejecución de los proyectos de aula. En relación al momento de la observación en que se encuentran las diferencias, los datos muestran un patrón de incremento significativo en los indicadores de actuación docente una vez finalizada la etapa de ejecución de los proyectos en aula, salvo el indicador guía el trabajo de los alumnos cuyo patrón es inverso, es decir, a medida que se desarrollaron los proyectos de aula los profesores guiaban cada vez menos a sus estudiantes.

TABLA 1. Resultados análisis de varianza, actuación docente en cada uno de los indicadores, respecto al inicio, proceso y fase final en la ejecución de los proyectos de aula.

\begin{tabular}{|c|c|c|c|c|}
\hline & Media & D.S. & $\mathrm{F}$ & $\begin{array}{c}\text { Contrastes } \\
\text { significativos }\end{array}$ \\
\hline Explicita objetivos de actividad & & & 6,035 & \\
\hline Inicio & 1,82 & 405 & & \\
\hline Proceso & 2,15 & ,689 & & \\
\hline Final & 2,64 & ,633 & & \\
\hline Evalúa conocimientos previos & & & $27,941^{* * *}$ & $1<2,3$ \\
\hline Inicio & 1,27 & ,647 & & \\
\hline Proceso & 2,54 &, 519 & & \\
\hline Final & 2,79 &, 426 & & \\
\hline Responde preguntas & & & 2,107 & \\
\hline Inicio & 2,18 &, 751 & & \\
\hline Proceso & 2,69 &, 480 & & \\
\hline Final & 2,57 & ,646 & & \\
\hline Hace preguntas de reflexión & & & $18,957 * * *$ & $1<2,3$ \\
\hline Inicio & 1,45 &, 522 & & \\
\hline Proceso & 2,62 &, 506 & & \\
\hline Final & 2,71 &, 611 & & \\
\hline Relaciona contenidos de economía entre asignaturas & & & $34,728 * * *$ & $1<2<3$ \\
\hline Inicio & 1,45 &, 522 & & \\
\hline Proceso & 2,46 &, 519 & & \\
\hline Final & 2,93 &, 267 & & \\
\hline Dirige el trabajo de los alumnos & & & $10,821 * * *$ & $1>2,3$ \\
\hline Inicio & 2,82 & ,603 & & \\
\hline Proceso & 2,54 &, 519 & & \\
\hline Final & 1,71 &, 726 & & \\
\hline Atiende dificultades & & & 2,640 & \\
\hline Inicio & 2,82 & ,405 & & \\
\hline Proceso & 2,69 & ,480 & & \\
\hline Final & 2,29 &, 825 & & \\
\hline Retroalimenta el desempeño & & & $40,094 * * *$ & $1<2<3$ \\
\hline Inicio & 1,18 & ,405 & & \\
\hline Proceso & 2,08 & ,494 & & \\
\hline Final & 2,79 &, 426 & & \\
\hline Utiliza metodologías cooperativas & & & $20,862 * * *$ & $1<2<3$ \\
\hline Inicio & 1,18 & 405 & & \\
\hline Proceso & 1,92 &, 277 & & \\
\hline Final & 2,57 & ,756 & & \\
\hline
\end{tabular}

Nota: $* * * \mathrm{p}<0,001$.

1 (inicio), 2 (proceso), 3 (final). 
Al analizar el efecto multivariado del momento de ejecución, establecimiento de pertenencia y asignaturas desarrolladas por cada profesor en relación a su impacto en los indicadores de actuación docente, los resultados señalan la obtención de efectos multivariados significativos para la variable momento de ejecución (Traza de Pillai $=1,607, F=4,085$, p < 0.005). Este resultado confirma los datos arrojados por el análisis de varianza presentado anteriormente. Interesa destacar que el análisis de los efectos univariados (ver Tabla 2) muestra que el indicador explica objetivos presenta diferencias significativas respecto al momento de ejecución de los proyectos $(F=8,631, \mathrm{p}<0,03)$, antecedente que en el análisis anterior resultó no ser estadísticamente significativo.

TABLA 2. Efectos univariados variable actuación docente respecto a momento de ejecución de los proyectos de aula.

\begin{tabular}{ll}
\hline & $\mathrm{F}$ \\
\hline Explicita objetivos & $8,631^{* *}$ \\
Evalúa conocimientos previos & $25,948^{* * *}$ \\
Responde preguntas & 2,674 \\
Hace preguntas de reflexión & $8,276^{* *}$ \\
Relaciona contenidos & $26,785^{* * *}$ \\
Guía el trabajo de los alumnos & $18,272^{* * *}$ \\
Atiende dificultades & 0,989 \\
Retroalimenta el desempeño & $48,300^{* * *}$ \\
Utiliza metodologías cooperativas & $14,165^{* * *}$ \\
\hline
\end{tabular}

$$
\text { Nota: } * * \mathrm{p}<0,05, * * * \mathrm{p}<0,001 \text {. }
$$

De esta forma, es rescatable del análisis multivariado que la actuación docente difiere entre los momentos de ejecución de los proyectos de aula mostrando un incremento progresivo en la efectividad docente, pero no difiere estadísticamente según el establecimiento de pertenencia ni la asignatura desarrollada por los profesores participantes lo cual indica el efecto compensatorio ejercido por el proceso de capacitación.

\section{DISCUSIÓN}

Los resultados obtenidos en esta investigación permiten concluir que el programa de formación en educación económica para docentes tiene efectos significativos en los niveles de Alfabetización Económica y actuación en aula de los profesores participantes.

Concordante con la literatura previa, los resultados confirman que los profesores participantes presentan una deficiente preparación en temas económicos vinculada no sólo con la falta de una formación sistemática en economía en su formación inicial, sino también con sus propios pre-conceptos y la forma cómo vivencian afectivamente el mundo 
económico (Hauwiller, 2001; Parkinson et al., 2004; Vargha, 2004; Walstad y Rebeck, 2001). En esta misma línea, los resultados demuestran que el proceso de entrenamiento y capacitación tuvo un importante impacto en el cambio de las metodologías pedagógicas, las que fueron ganando en calidad y organización en la medida que avanzó el proceso de desarrollo de los proyectos en aula. Ello es concordante con otras experiencias de formación de profesores para la educación económica en contextos internacionales (Kourilsky, 1996; Moos, 2007; Parkinson et al., 2004; Vargha, 2004; Walstad y Allgood, 1999; Walstad y Rebeck, 2001).

Un aspecto clave del proceso de capacitación lo constituyó la evaluación inicial del nivel de alfabetización económica de los participantes que permitió una retroalimentación preliminar en torno a sus competencias en este ámbito. Esta constatación permitió además revisar la propuesta teórica del proceso de capacitación y focalizar la intervención en el aprendizaje de los conceptos claves que aparecían con menor nivel de apropiación. Otro aspecto relevante radica en la emergencia de un proceso de reflexión sobre los propios comportamientos económicos y creencias en torno al consumo y la propia actuación en este ámbito, ello facilitó la apertura hacia una evaluación más cercana a la experiencia de los escolares y la necesidad de una intervención pedagógica orientada a estimular un comportamiento económico más reflexivo.

La variedad de procesos vividos en aula, la necesidad de coordinar los intereses docentes con la experiencia y necesidades de los estudiantes, así como la incorporación de metodologías pedagógicas más acordes a la construcción de aprendizajes significativos, demostró un importante impacto en el resultado obtenido y en el cambio del escenario docente que los profesores fueron capaces de desplegar. Por otra parte, si bien en este trabajo no se da cuenta del nivel de aprendizaje logrado por los estudiantes, aspecto que compete a otra etapa del proyecto, es posible hipotetizar que el cambio docente efectivamente impactaría la calidad de los aprendizajes y autonomía de los escolares, ello se vincula a la constatación de disminución del trabajo guiado por el profesor acompañado de un aumento en las estrategias pedagógicas que potenciaban la autonomía. En este punto, cobró especial relevancia la incorporación del trabajo cooperativo y nuevas metodologías de evaluación, resultado que concuerda con lo informado por Parkinson et al., (2004) y Moos (2007), constituyéndose adicionalmente en una herramienta para consolidar comportamientos ciudadanos en un espacio pedagógico democrático (Comisión de Formación Ciudadana, 2004).

Así la constatación de la falta de diferencias en la actuación de los profesores de distintos sectores de aprendizaje (Estudio y Comprensión del Medio Social, Matemáticas y Lenguaje y Comunicación) refuerza la importancia de una aproximación interdisciplinaria a la enseñanza de conceptos económicos (Denegri y Martínez, 2003), así como la influencia compensadora del proceso de capacitación inicial.

Tal como se señaló, un aspecto no abordado en este trabajo es el rendimiento real alcanzado por los estudiantes, aspecto que deberá ser incorporado en la próxima etapa del proyecto. A la vez resulta relevante incorporar un proceso de seguimiento de la actuación docente una vez terminada la etapa experimental del proyecto "Yo y la economía" de modo de evaluar a mediano y largo plazo la permanencia de los cambios observados, aspecto que requiere de investigaciones posteriores. 
Finalmente, si bien la muestra de profesores participantes fue relativamente pequeña, especialmente por la complejidad del diseño de formación y seguimiento en aula, el proceso de selección de la muestra así como los resultados obtenidos parecen ser generalizables para la aplicación masiva del programa "Yo y la economía", lo que constituye un aporte a un ámbito tan descuidado y a la vez vital para el desarrollo ciudadano, personal y familiar de los educandos.

\section{CONCLUSIÓN}

La presente investigación se propuso (a) describir el nivel de alfabetización económica al iniciar el programa de capacitación, (b) comparar los cambios en los niveles de alfabetización económica posterior a la capacitación, y (c) comparar los cambios en la actuación docente al inicio, durante y al final de la aplicación experimental del programa de educación económica Yo y la Economía.

En relación al primer objetivo es preciso concluir que el nivel de alfabetización económica inicial consideró un rendimiento de un $44 \%$ con una media de 10,285 (d.s=2,61), en tanto que la segunda evaluación, correspondiente al segundo objetivo, consideró un rendimiento de un $77,25 \%$ con una media de $17,77($ d.s $=2,45)$.

Finalmente, respecto al tercer objetivo los resultados indican diferencias significativas respecto al momento de desarrollo de los proyectos de aula en 6 de los XX indicadores, mostrando un patrón de incremento significativo en los indicadores de actuación docente una vez finalizada la etapa de ejecución de los proyectos en aula, a excepción del indicador guía el trabajo de los alumnos, cuyo patrón es inverso debido al contenido que evalúa.

\section{NOTA DE RECONOCIMIENTO}

Esta investigación es parte de un programa de mayor envergadura financiado por el Consejo de Ciencia y Tecnología de Chile (CONICYT) a través de los proyectos FONDECYT (Fondo Nacional de Desarrollo Científico y Tecnológico) N. ${ }^{\circ} 1060303$ "Yo y la economía": Diseño, Aplicación y Evaluación de impacto de un Programa de Educación Económica en escolares de $6 .^{\text {to }}$ año básico de Temuco; y N. ${ }^{\circ} 1090179$ “Alfabetización económica y Patrones de consumo y endeudamiento en estudiantes de Pedagogía: Hacia un modelo explicativo".

\section{REFERENCIAS BIBLIOGRÁFICAS}

1. Alemayehu, T. (2007). Economic education. A requirement for building a free market economy and a liberal democracy in Etiopía. International Business and Economic Research Journal, 6, 9, 85-95.

2. Comisión Formación Ciudadana (2004). Informe Comisión Ciudadana. Chile.

3. Cumbre extraordinaria de las Américas (2004). Declaración de Nuevo León. En Documentos Oficiales del proceso de Cumbres de las Américas. Washington. 
4. Denegri, M. (2005). Proyectos de aula interdisciplinarios y reprofesionalización de profesores: un modelo de capacitación. Estudios Pedagógicos, 31, (1), 35-50.

5. Denegri, M., Palavecinos, M., Ripoll, M. y Yáñez, V. (1999). Caracterización psicológica del consumidor de la IX Región. En Denegri, M., Iturra, R., Palavecinos, M. y Ripoll, M. (eds.). Consumir para vivir y no vivir para consumir. Chile: Ediciones Universidad de La Frontera.

6. Denegri, M. y Martínez, G. (2003). Proyectos de aula interdisciplinarios; un aporte constructivista a la reprofesionalización de profesores. Revista Profissao Docente Online Universidade de Uberaba, Set/Dez. Extraído desde, http://www.uniube.br/ propep/mestrado/revista/vol03/09/art01.htm

7. Denegri, M. y Palavecinos, M. (2003). Género y alfabetización económica ¿oportunidades de desarrollo o nuevos caminos para discriminación? Revista Psicología desde el Caribe, 12, 76-97.

8. Denegri, M., Palavecinos, M. y Gempp, R. (2005). Informe Final Proyecto Fondecyt N. ${ }^{o}$ 1030271. Santiago: Fondecyt.

9. Denegri, M.; Del Valle, C.; Gempp, R. y Lara, M. (2006a). Educación Económica en la escuela. Hacia una propuesta de intervención. Estudios Pedagógicos, 32 (2).

10. Denegri, M.; Gempp, R.; Del Valle, C.; Etchebarne, S. y González, Y. (2006b). El aporte de la psicología educacional a las propuestas de educación económica: los temas claves. Revista de Psicología, 15 (2), 77-94.

11. Domper, M. (2004). ¿Qué aprenden de economía nuestros niños en el colegio? Serie Informe Económico N. ${ }^{\circ} 151$. Instituto Libertad y Desarrollo. Chile.

12. Gempp, R., Denegri, M., Caripán, N. y Catalán, V. (2007). Desarrollo del Test de Alfabetización Económica para Adultos TAE-A-25. Revista Interamericana de Psicología, 41 (2).

13. Georgius, G. (1996). Pre-College Economics Instruction and Outcome Scores. Journal of Business and Economic Studies, 9(1), 65-82.

14. Gleason, J. y Van Scyoc, L. (1995). A report on the economic literacy of adults. Journal of Economic Education, 26, 3, 206-210.

15. Hauwiller, J. (2001). Redesigning Economic Education Workshops. Education, 103 (4), 383-386.

16. Johnston, B. y Maguire, B. (2004). Adults numeracy: policy and practice in global context of lifelong learning. Working Paper. ALM, Research Forum, Denmark.

17. Kourilsky, M. (1996). Generative Teaching and Personality Characteristics of Student Teachers. Teaching and Teacher Education, 12 (4), 355-363.

18. Laney, J. (1993). Economics for Elementary school students: Research-supported principles of teaching and learning that guide classroom practice. Social Studies, 99-103. 
19. Laney, J. (1999). A Sample Lesson in Economics for Primary Students. How Cooperative and Mastery Learning Methods can Enhance Social Studies Teaching. Social Studies, 152-158.

20. Lusardi, A. y Mitchelli, O. (2007). Financial literacy and retirement preparedness. Evidence and implication for financial Education. Business Economic, 42(1), 35.

21. Machinea, J. (2007). Desafíos económicos y sociales de América Latina y el Caribe: algunas consideraciones. En Hacia la Quinta Cumbre de las Américas. Desafíos Regionales. Washington: Organización de los Estados Americanos.

22. Mandell, L. (2008). Financial literacy of high school students. Handbook of Consumer Finance Research. 183-183.

23. Medina, M., Méndez, G. y Pérez, C. (1998). Actitudes hacia el endeudamiento en estudiantes universitarios. Tesis de Licenciatura en Psicología no publicada, Universidad de La Frontera, Temuco, Chile.

24. Moore-Shay, E. y Berchmans, B. (1996). The role of the family environment in the development of shared consumption values: an intergenerational study, Advances in Consumer Research, 23, 484-490.

25. Moos, M. (2007). The use of cooperative learning in economic in the further education and training phase in the free state province. Thesis Philosophie Doctor. University of the Free State Bloemfontein, South Africa.

26. Mori, A. (2001). Money in the contemporary Family. Nestle Family Monitor, 20.

27. National Council on Economic Education (1997). Voluntary National Content Standards in Economic. National Council on Economic Education: New York.

28. Orton, L. (2007). Financial literacy: lesson fron internacional experience. Canadian Policy Research Network, 1-33.

29. Page, C. y Ridgway, N. (2001). The impact consumer environments on consumption patterns on children from disparate socioeconomic backgrounds, Journal of Consumer Marketing, 18, 21-40.

30. Parkinson, K., Sorgman, M. y Millar, Ch. (2004). Intersection of teacher perception and state economic standarss. Journal of Economic and Economic Education Research, 5, (2), 37-49.

31. Rose, G. (1999). Consumer socialization, parental style, and developmental timetables in the United States and Japan. Journal of Marketing, 63, 105-119.

32. Schug, M. (1996). Introducing Children to Economic Reasoning: Some Beginning Lesson. The Social Studies, 87 (3).

33. Schug, M. y Hagedorn, E. (2005). The Money Savvy PigTM Goes to the Big City: Testing the Effectiveness of an Economics Curriculum four Young Children. The Social Studies, 68-71. 
34. Sosin, K., Dick, J. y Lynn, M. (1997). Determinants of Achievement of Economics Concepts by Elementary School Students. Journal of Economic Education, 100.

35. Varcoe, K. y Fitch P. (2003). Money talks- a program to improve financial literacy of teens. International Journal of Consumer Studies, 27 (3), 218-251.

36. Vargha, L. (2004). Buyer Beware! Economics Activities for Middle School Students. Social Studies, 27-32.

37. Volpe, R., Haiyang, Ch. y Sheen, L. (2006). An analysis of personal finance topics and the level of knowledge possessed by working adults. Financial Services Review, 15, 81-98.

38. Walstad, W. y Allgood, S. (1999). What do college know about economics? American Economic Review, 89, 350-354.

39. Walstad, W. y Rebeck, K. (2001). Teacher and Student Economic Understanding in Transition Economies. Journal of Economic Education, 58-67.

40. Wentland, D. (2004). A Guide for Determining which Teaching Methodology to Utilize in Economic Education: Trying to Improve how Economic Information is Communicated to Students, Education, 124, 640-648. 